\title{
A generalized burke-schumann formula- tion for hydrogen-oxygen diffusion flames maintaining partial equilibrium of the shuffle reactions
}

\author{
ANTONIO L. SÁNCHEZ , AMABLE LIÑÁN'
}

FORMAN A. WILLIAMS

Under a wide range of conditions of ambient pressures, temperatures, dilutions and strain rates, nonpremixed combustion in hydrogen-oxygen systems maintaits partial equilibrium of the fout two-body chain-carrying reactions while experiencing finite rates of the three-body radical-recombination reactions $\mathrm{H}+\mathrm{O}_{2}+\mathrm{M} \rightarrow \mathrm{HO}_{2}+\mathrm{M}$ and $\mathrm{H}+\mathrm{H}+\mathrm{M} \rightarrow \mathrm{H}_{2}+\mathrm{M}$. There then exists a three-step reduced mechanism, with $\mathrm{H}$ as the only intermediate species and concentrations of the radicals $\mathrm{O}, \mathrm{OH}$ and $\mathrm{HO}_{2}$ related to that of $\mathrm{H}$ through steady states. The conservation equations corresponding to this chemical description are formulated here in terms of generalized coupling functions that account for species diffusivities that differ from the thermal diffusivity, providing a set of equations that describe the flame structure for strain conditions ranging from near extinction to weakly strained flames. As a model example, the formulation is applied to the analysis of flame development in the hydrogen-air laminar mixing layer with free-stream temperatures above the crossover temperature corresponding to the second explosion limit. The formulation can be used for many other model problems as well as for computational studies of nonpremixed combustion in complex configurations involving both laminar and turbulent flows.

\section{INTRODUCTION}

Reactive flows are complicated phenomena for which the interactions between the fluid dynamics and the chemistry play a fundamental role. 
Although the limit of infinitely large Damköhler numbers, in which all chemical processes are assumed to be infinitely fast, is useful in describing very weakly strained flows, finite-rate effects are significant under most strain conditions and must be taken into account if accurate descriptions are to be obtained. In general, different chemical processes exhibit very different time scales at the temperatures present in reaction zones, with radical-shuffling reactions typically being the fastest. Seeking simplifications that account for the disparity of the different time scales through steadystate assumptions for the radicals and through partial-equilibrium assumptions for the fast reactions has been common practice in recent years (Janicka and Kollmann, 1979), leading to reduced chemical-kinetic mechanisms that potentially can be used in numerical calculations of complex reactive flows. Following our previous work on counterflow flames (Sánchez et al., 1995), we present here a general three-step reduced mechanism for hydrogen-oxygen nonpremixed combustion with infinitely fast radicalshuffling reactions and finite recombination rates that is seen to apply under a wide range of flowfield conditions. The generalized coupling-function formalism originally developed by Liñán and Williams (1993) is employed to transform the corresponding conservation equations, yielding a formulation that circumvents the stiffness of the fast radical-shuffling reactions, while accounting for the different values of the thermal and species diffusivities.

Approaches to the analytical study of hydrogen-air diffusion flames with reduced chemistry have been developed in recent years (Gutheil and Williams, 1990; Tangirala et al., 1991; Balakrishnan et al., 1992; Lee and Chung, 1994; Sánchez et al., 1995), yielding different descriptions for different combustion regimes. For instance, the asymptotic structure of the counterflow flame for strain times much larger than the characteristic time for radical recombinations is given by Sánchez et al. (1995), who found that under those strain conditions the reactants can coexist only within a thin reaction zone, where the fast shuffle reactions maintain partial equilibrium, separating two radical-free equilibrium regions. As the strain rate was increased, the reaction layer was seen to become thicker, and radicals were seen to spread over a wider part of the counterflow mixing layer, causing the results of the analysis to lose accuracy with increasing strain rates. It was also observed that, as the temperature drops below the Burke-Schumann peak temperature through finite-rate effects, the partial equilibrium of the shuffle reactions becomes increasingly pronounced, eventually leading to a threelayer inner structure within the reaction zone, with reactants coexisting only in a very thin radical-production layer. 
These ideas can be extended naturally to describe more general strain conditions by noticing that the altered character of the partial-equilibrium approximation prevents the reactants from coexisting, yielding a BurkeSchumann-like characteristic flowfield structure in which regions of fuel and oxidizer and separated by thin radical-production layers, resulting in radicals being distributed throughout the flow field. Heat release through radical recombinations is permitted everywhere, enabling diffusion flames with strain times of the order of the radical-recombination time to be described. In the limit considered here, the concentration gradients of the different species will be seen to exhibit discontinuities at the radical-production layer. This singular character will be overcome by formulating the conservation equations in terms of generalized coupling functions, similar to those previously employed by Liñán and Williams (1993) in the study of flows with infinitely fast one-step chemistry.

The reduced mechanism resulting from these ideas involves partial equilibrium of the shuffle reactions and finite rates of the three-body recombinations. Similar chemistry was employed by Janicka and Kollmann (1979), who included the steps $\mathrm{H}+\mathrm{OH}+\mathrm{M} \rightarrow \mathrm{H}_{2} \mathrm{O}+\mathrm{M}$ and $\mathrm{H}+\mathrm{O}+\mathrm{M} \rightarrow \mathrm{OH}$ $+\mathrm{M}$, which we find to be unimportant, but neglected the recombination step $\mathrm{H}+\mathrm{O}_{2}+\mathrm{M} \rightarrow \mathrm{HO}_{2}+\mathrm{M}$, which is dominant on the oxygen side. Janicka and Kollmann were concerned with turbulent diffusion flames, and this same chemistry was adopted in a number of later studies of such problems (Bilger, 1989). None of these studies employed the present type of general formulation, which can be used to describe turbulent diffusion flames without necessitating treatment of chemistry with widely different times scales or implementation of front-tracking techniques or interface approximations.

As an example, we shall apply the proposed general formulation to the description of flame development in the hydrogen-air mixing layer with freestream temperatures above the crossover temperature corresponding to the second explosion limit, at which the rate of the main branching step $\mathrm{H}+\mathrm{O}_{2} \rightarrow \mathrm{OH}+\mathrm{O}$ equals that of the main recombination step $\mathrm{H}+\mathrm{O}_{2}+$ $\mathrm{M} \rightarrow \mathrm{HO}_{2}+\mathrm{M}$ (Lewis and Von Elbe, 1987; Kreutz and Law, 1996). This flow is relevant, for instance, in high-speed air-breathing propulsion devices that incorporate injection of hydrogen into a hot stream of air, a process that leads to spontaneous ignition downstream of the injector, where a diffusion flame forms. Although the analytical study of nonpremixed reactive mixing layers with a one-step irreversible reaction adopted for the chemistry description was completed twenty years ago by Liñan and Crespo (1976), who employed the large value of the activation energy typical of combustion 
processes as a large parameter for their asymptotic analysis, mixing-layer investigations with realistic chemistry for hydrogen-air systems have been conducted only recently. Most of the work has been numerical (Figueira Da Silva et al., 1993; Ju and Niioka, 1994), with asymptotic analyses being restricted to the investigation of chain-branching induction regions (Treviño and Liñan, 1995; Sánchez et al., 1997). The extension of these analyses to describe the heat-release process that occurs downstream these induction regions is presented here.

\section{REDUCED KINETIC MECHANISM}

An eight-step elementary mechanism adequate for the description of hydrogen diffusion flames, selected from previous studies (Gutheil and Williams, 1990; Gutheil et al., 1993; Sánchez et al., 1995), is composed of the two three-body recombination reactions

$$
\mathrm{H}+\mathrm{O}_{2}+\mathrm{M} \stackrel{1 f}{\rightarrow} \mathrm{HO}_{2}+\mathrm{M}
$$

and

$$
\mathrm{H}+\mathrm{H}+\mathrm{M} \stackrel{2 f}{\rightarrow} \mathrm{H}_{2}+\mathrm{M},
$$

along with the four shuffle reactions

$$
\begin{gathered}
\mathrm{H}+\mathrm{O}_{2} \stackrel{3}{\rightleftharpoons} \mathrm{OH}+\mathrm{O}, \\
\mathrm{O}+\mathrm{H}_{2} \stackrel{4}{\rightleftharpoons} \mathrm{OH}+\mathrm{H}, \\
\mathrm{OH}+\mathrm{H}_{2} \stackrel{5}{\rightleftharpoons} \mathrm{H}_{2} \mathrm{O}+\mathrm{H} \\
\mathrm{OH}+\mathrm{OH} \stackrel{\stackrel{6}{\rightleftharpoons}}{\rightleftharpoons} \mathrm{H}_{2} \mathrm{O}+\mathrm{O},
\end{gathered}
$$

and

and the two hydroperoxyl-consuming reactions

$$
\mathrm{HO}_{2}+\mathrm{H} \stackrel{7 f}{\rightarrow} \mathrm{H}_{2}+\mathrm{O}_{2}
$$

and

$$
\mathrm{HO}_{2}+\mathrm{H} \stackrel{8 f}{\rightarrow} \mathrm{OH}+\mathrm{OH} \text {. }
$$

Updated values of the reaction-rate constants corresponding to the above mechanism can be found elsewhere (Balakrishnan and Williams, 1994). Inderiving this simplified scheme we have taken into account that in diffusion 
flames recombination steps other than reactions $1 f$ and $2 f$ are unimportant, that the concentration of $\mathrm{H}_{2} \mathrm{O}_{2}$ is negligible and that $\mathrm{HO}_{2}$ consumption by the reverse of step 1 and by other two-body steps is small compared to that of reactions $7 f$ and $8 f$ (Gutheil and Williams, 1990; Gutheil et al., 1993).

The partial-equilibrium approximation for the shuffle reactions provides three independent algebraic relationships among the concentrations of the reactive species, which can be expressed for instance as

$$
\begin{aligned}
& X_{\mathrm{H}}=K_{\mathrm{H}} X_{\mathrm{H}_{2}}^{3 / 2} X_{\mathrm{O}_{2}}^{1 / 2} / X_{\mathrm{H}_{2} \mathrm{O}}, \\
& X_{\mathrm{OH}}=K_{\mathrm{OH}} X_{\mathrm{H}_{2}}^{1 / 2} X_{\mathrm{O}_{2}}^{1 / 2} \\
& X_{\mathrm{O}}=K_{\mathrm{O}} X_{\mathrm{H}_{2}} X_{\mathrm{O}_{2}} / X_{\mathrm{H}_{2} \mathrm{O}},
\end{aligned}
$$

and

where $X_{i}$ is the mole fraction of species $i$ and $K_{\mathrm{H}}=1.27 \exp (2997 / T)$, $K_{\mathrm{OH}}=5.97 \exp (-4696 / T)$ and $K_{\mathrm{O}}=3.41 \exp (-704 / T)$ are equilibrium constants related to the equilibrium constants of reactions 3-5 (Tangirala et al., 1991) through $K_{\mathrm{H}}=K_{3}^{1 / 2} K_{4}^{1 / 2} K_{5}, K_{\mathrm{OH}}=K_{3}^{1 / 2} K_{4}^{1 / 2}$ and $K_{\mathrm{O}}=K_{3} K_{5}$, whose temperature dependences are exhibited in Figure 1. These relationships can be simplified considerably by taking into account that for temperatures of practical interest $K_{\mathrm{H}} \gg 1$ and $K_{\mathrm{OH}} \ll 1$, with $K_{\mathrm{O}}$ and $K_{\mathrm{H}} K_{\mathrm{OH}}$ being both quantities of order unity, as can be inferred from Figure 1. Introducing

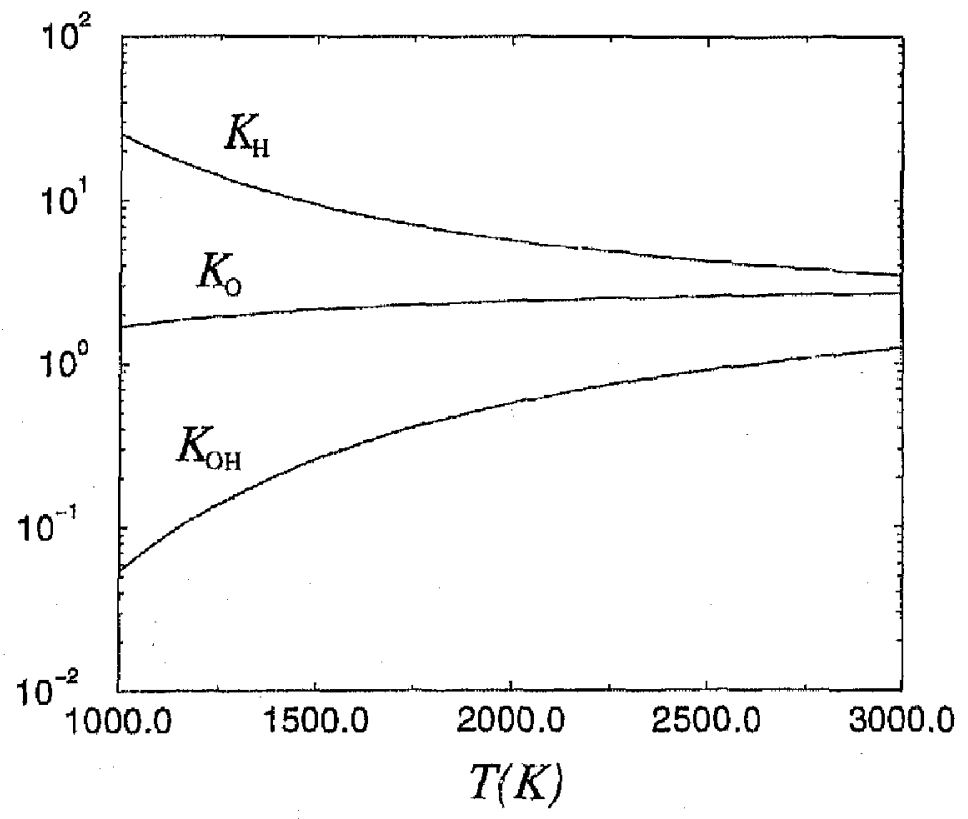

FIGURE 1 The variation of the constants $K_{\mathrm{H}}, K_{\mathrm{OH}}$ and $K_{\mathrm{O}}$ with temperature. 
these scalings into Equation 1 and anticipating that both $X_{\mathrm{H}}$ and $X_{\mathrm{H}_{2} \mathrm{O}}$ are of order unity yields the Burke-Schumann condition $X_{\mathrm{O}_{2}} X_{\mathrm{H}_{2}}=0$; in other words, in the realistic limit $K_{\mathrm{H}} \gg 1$ the partial-equilibrium approximation of the shuffle reactions implies that the reactants cannot coexist, leading to a flow field in which the oxidizer and fuel regions are separated by infinitesimally thin radical-production layers. Observation of Equation 1 reveals that the reactants coexist in this radical-production layer with characteristic mole fractions of order $K_{\mathrm{H}}^{-1 / 2}$, while over previous work (Sánchez et al., 1995 ) indicates that outside this thin layer the $\mathrm{H}_{2}$ concentration is of order $K_{\mathrm{H}}^{-2 / 3}$ on the oxygen side, and the $\mathrm{O}_{2}$ concentration is of order $K_{\mathrm{H}}^{-2}$ on the hydrogen side. Also, since $K_{\mathrm{OH}}$ is of order $K_{\mathrm{H}}^{-1}$ and $K_{\mathrm{O}}$ is of order unity, inspection of Equations 2 and 3 shows that the concentrations of $\mathrm{OH}$ and $O$ are small everywhere, with characteristic values of orders $K_{\mathrm{H}}^{-4 / 3}$ and $K_{\mathrm{H}}^{-2 / 3}$, respectively, on the oxidizer side and of order $K_{\mathrm{H}}^{-2}$ on the fuel side. These values are sufficiently small that, because of the partial equilibria of the reactions $\mathrm{H}+\mathrm{O}_{2} \stackrel{3}{\rightleftharpoons} \mathrm{OH}+\mathrm{O}$ and $\mathrm{H}_{2}+\mathrm{OH} \stackrel{5}{\rightleftharpoons} \mathrm{H}_{2} \mathrm{O}+\mathrm{H}$ at the radicalproduction layer, $\mathrm{OH}$ and $\mathrm{O}$ radicals maintain steady states everywhere, giving small concentrations that can be neglected in the first approximation for the description of the flame. These steady states are less accurate for ignition (Balakrishnan et al., 1995), emphasizing that the present considerations apply well only after the diffusion flame is established. With the steady-state assumptions for $\mathrm{OH}$ and $\mathrm{O}$ incorporated, the partial-equilibrium approximation of the shuffle reactions yields a radical-production step $3 \mathrm{H}_{2}+\mathrm{O}_{2} \stackrel{\mathrm{I}}{\rightarrow} 2 \mathrm{H}_{2} \mathrm{O}+2 \mathrm{H}$ (Sánchez et al., 1995), which is infinitely fast in the limit $K_{\mathrm{H}} \gg 1$ considered here. The reactants reach by diffusion the thin radical-production layers in stoichiometric proportions dictated by this global reaction I. Because of the different stoichiometric coefficient from that of the original overall reaction $2 \mathrm{H}_{2}+\mathrm{O}_{2} \rightarrow 2 \mathrm{H}_{2} \mathrm{O}$, this layer is displaced to the fuel side in conventional mixture-fraction space.

The $\mathrm{H}$ atoms produced in the radical-production layer are recombined outside that layer by reactions $\mathrm{H}+\mathrm{O}_{2}+\mathrm{M} \stackrel{15}{\rightarrow} \mathrm{HO}_{2}+\mathrm{M}$ and $\mathrm{H}+\mathrm{H}+$ $\mathrm{M} \stackrel{2 S}{\rightarrow} \mathrm{H}_{2}+\mathrm{M}$. The hydroperoxyl radicals produced by reaction $1 f$ are consumed rapidly by the fast reactions $\mathrm{HO}_{2}+\mathrm{H}^{7 f} \rightarrow \mathrm{H}_{2}+\mathrm{O}_{2}$ and $\mathrm{HO}_{2}+\mathrm{H} \stackrel{8 f}{\rightarrow}$ $\mathrm{OH}+\mathrm{OH}$ and can be assumed to maintain steady state everywhere. We denote the rate of step $i$ by $\omega_{i}$ and the corresponding specific reaction-rate constant by $k_{i}$. Evaluation of $k_{7 f}$ and $k_{8 f}$ reveals that most of the $\mathrm{HO}_{2}$ radicals produced are consumed by the chain-carrying reaction $8 f$, partially attenuating, therefore, the chain-terminating effect of reaction $1 f$. The fraction of hydroperoxyl radicals consumed by the chain-breaking reaction $7 f$ is $\alpha=k_{7 f} /\left(k_{7 f}+k_{8 f}\right) \simeq 1 / 6$, giving a global chain-breaking reaction 
$\mathrm{H}+\mathrm{H} \rightarrow \mathrm{H}_{2}$ with rate $\alpha \omega_{1 f}$, as can be obtained by adding reactions $1 f$ and 7f. Similarly, combining reactions $1 f$ and $8 f$ with reaction $\mathrm{H}_{2}+\mathrm{OH} \rightarrow$ $\mathrm{H}_{2} \mathrm{O}+\mathrm{H}$, corresponding to partial equilibrium of reaction 5, yields $2 \mathrm{H}_{2}+$ $\mathrm{O}_{2} \rightarrow 2 \mathrm{H}_{2} \mathrm{O}$ with rate $(1-\alpha) \omega_{1 f}$ for the global step associated with the chain-carrying path.

From these results, the overall kinetic mechanism simplifies to the infinitely fast radical-production step

$$
3 \mathrm{H}_{2}+\mathrm{O}_{2} \stackrel{\mathrm{l}}{\rightarrow} 2 \mathrm{H}_{2} \mathrm{O}+2 \mathrm{H}
$$

together with the two recombination steps

$$
\begin{gathered}
\mathrm{H}+\mathrm{H} \stackrel{\text { II }}{\rightarrow} \mathrm{H}_{2} \\
2 \mathrm{H}_{2}+\mathrm{O}_{2} \stackrel{\text { III }}{\rightarrow} 2 \mathrm{H}_{2} \mathrm{O}
\end{gathered}
$$

with rates $\alpha \omega_{1 f}+\omega_{2 f}$ and $(1-\alpha) \omega_{1 f}$, respectively. The rate constant of the recombination reactions $1 f$ and $2 f$ are of the form $k_{j}=A_{j} T^{n_{j}}$. Updated values of the different reaction-rate parameters, for units $\mathrm{mol} / \mathrm{cm}^{3}, \mathrm{~s}^{-1}$ and $\mathrm{K}$, are (Balakrishnan and Williams, 1994) $A_{1 f}=6.76 \times 10^{19}, A_{2 f}=1.80 \times 10^{18}$, $n_{1 f}=-1.42$ and $n_{2 f}=-1$. The third-body Chaperon efficiencies of $\mathrm{O}_{2}, \mathrm{H}_{2}$, $\mathrm{H}_{2} \mathrm{O}$ and $\mathrm{N}_{2}$ are, respectively, 1.0, 2.5. 12.0 and 1.0 for reaction $1 f$ and 0.4 , $1.0,6.5$ and 0.4 for reaction $2 f$, Note that the rate $\omega_{1 f}$ is unimportant on the fuel side of the flow field, where the $\mathrm{O}_{2}$ concentration is negligible. On the oxidizer side, where both recombination rates $\omega_{1 f}$ and $\omega_{2 f}$ are equally important, $\mathrm{H}_{2}$ maintains steady state because of the partial equilibrium of the shuffle reactions, i.e., excess fuel generated by reaction II is consumed at an infinite rate by step I.

Limitations to the applicability of this mechanism for the description of nonpremixed hydrogen-oxygen combustion can be inferred by examining the underlying assumptions involved in its derivation. First, it is assumed that the shuffe reactions maintain partial equilibrium everywhere. Bearing in mind that the characteristic local strain time associated with the flow must be on the order of or larger than the characteristic time of radical recombination for a flame to exist, we see that this condition reduces to the requirement that the temperature be sufficiently high above the crossover temperature of the rates of the branching and recombination steps, so that the characteristic time associated with the shuffle reactions is considerably smaller than that of radical recombinations and, consequently, also smaller than the local strain time. Therefore, extinction events, which are in general 
associated with small strain times and with temperatures relatively close to crossover, can be addressed only partially with the above mechanism, which nevertheless does provide a simplified criterion for extinction to occur, as shown below. On the other hand, it is assumed that the equilibrium constants of the shuffle reactions are such that $K_{\mathrm{H}} \gg 1$, leading to the BurkeSchumann condition $X_{\mathrm{O}_{2}} X_{\mathrm{H}_{2}}=0$, as explained above. While this condition holds over a wide range of temperatures of practical interest, observation of Figure 1 indicates that such an assumption becomes progressively inaccurate as the temperature increases. For instance, at the high temperatures typical of very weakly strained hydrogen-air diffusion fiames $(T \sim 2600)$, the partial-equilibrium condition of the shuffe reactions no longer implies that $X_{\mathrm{O}_{2}} X_{\mathrm{H}_{2}}=0$. Solution for structures of such robust flames must be add ressed by employing finite values for the equilibrium constants in Equations 1-3 as was done by Sánchez et al. (1995), who showed how the partial-equilibrium assumption for the shuffle reactions leads to the present Burke-Schumann condition as the strain time decreases. It is also worth pointing out that for even higher temperatures, such as those achieved when air is replaced by pure oxygen or when the reactants arc pre-heated sufficiently, the effect of the dissociation reactions can become significant, leading to equilibrium broadening, a behavior that cannot be captured with the present mechanism.

\section{GENERAL FORMULATION FOR $K_{H}=\infty$}

The problem of mixing and reaction in nonpremixed environments requires integration of the continuity and momentum equations, together with the species conservation equation

$$
\rho \frac{D Y_{i}}{D t}-\nabla \cdot\left(\frac{\mu}{S_{i}} \nabla Y_{i}\right)=w_{i}
$$

and energy conservation equation

$$
\rho \frac{D \theta}{D t}-\nabla \cdot\left(\frac{\mu}{P_{r}} \nabla \theta\right)=-\sum_{i}^{N} \frac{h_{i}^{0}}{c_{p} T_{\mathrm{rel}}} w_{i}
$$

where $D() / D t$ denotes the substantial derivative and $N$ is the number of reactive species present in the system. In this formulation, $\rho, \mu$ and 
$\theta=T / T_{\text {ref }}$ are the density, viscosity and nondimensional temperature of the mixture, with $T_{\text {ref }}$ being an arbitrary reference temperature. The mass fraction, mass rate of production and enthalpy of formation of species $i$ are denoted by $Y_{i}, w_{i}$ and $h_{i}^{\prime}$, respectively. In writing Equation 4, a Fickian description is adopted for the diffusion velocities. Because of the presence of significant amounts of $\mathrm{H}$ and $\mathrm{H}_{2}$, non-Fickian diffusion (Sánchez et al., 1996a) and thermal diffusion (Balakrishnan et al., 1995) may have a nonnegligible effect under some conditions, and should be incorporated in the formulation for increased accuracy. A low-Mach-number approximation with constant specific heat at constant pressure $c_{p}$ has been employed in deriving Equation 5, where unsteady pressure variations have also been neglected. Alternatively, thermal enthalpy can replace $\theta$ in writing the energy conservation equation, yielding an equation analogous to Equation 5 if one neglects the effect of the differences of the specific heat at constant pressure of each species from the mean $c_{p}$ (Sánchez et al., 1995), thereby enabling the temperature variation of $c_{p}$ to be taken into account in the formulation. Although such alternative formulation seems worth investigating for increased accuracy, we choose for simplicity 0 as an integration variable in the present paper. We shall also assume that the nondimensional transport numbers that appear above, i.e., the Schmidt number of species $i$, $S_{i}=\mu /\left(\rho D_{i}\right)$, and the Prandtl number, $\operatorname{Pr}=\mu c_{p} / \lambda$, are constant. Here, $\lambda$ and $D_{i}$ denote, respectively, the thermal conductivity and the binary diffusion coefficient of species $i$. The above equations, supplemented with the quasiisobaric form of the ideal gas law $\rho T \Sigma_{i}^{N} Y_{i} / W_{i}=$ constant, where $W_{i}$ represents the molecular weight of species $i$, must be integrated with appropriate initial and boundary conditions.

As previously mentioned, the altered character of the partial equilibrium of the shuffle reactions causes radical production to be confined within infinitesimally thin surfaces, where reactants, which cannot coexist outside these surfaces, meet by diffusion in ratios dictated by the infinitely fast step $3 \mathrm{H}_{2}+\mathrm{O}_{2} \rightarrow 2 \mathrm{H}_{2} \mathrm{O}+2 \mathrm{H}$. Because of the infinite rate of this reaction, the gradients of the different species concentrations exhibit jumps across the radical-production layers, whose location must be obtained as part of the solution, yielding a complicated problem similar to that encountered with one-step chemistry in the limit of infinitely large Damköhler numbers. Burke and Schumann (1928) indicated how the singular character of the solution can be overcome in the limit of equal reactant diffusivities (unity Lewis numbers) by introduction of conserved functions, the coupling functions (Williams, 1985), that behave like passive scalars. It is also possible to extend this analysis to configurations with non-unity Lewis numbers as 
shown by Liñán and Williams (1993), who developed a general formulation that has been utilized in the study of hydrogen-air counterflow flames (Sánchez et al., 1995) and elsewhere (Liñán et al., 1994). Following these previous ideas, we derive now the generalized coupling functions and associated conservation equations corresponding to the reduced-chemistry description presented above. Attention is restricted to problems in which the reactants are supplied by two independent strearns, with dilution by nitrogen permitted in both streams. The boundary conditions for Equations 4 and 5 associated with the resulting configuration involve the temperature and reactant mass fractions of the fuel and oxidizer streams, identified hereafter by the subscripts $f$ and $o$.

Equations 4 and 5 can be combined linearly to eliminate the infinitely large terms associated with step I, yielding

$$
\begin{gathered}
L_{\mathrm{H}_{2}}\left(s y_{\mathrm{H}_{2}}\right)-L_{\mathrm{O}_{2}}\left(3 y_{\mathrm{O}_{2}}\right)=\left(W_{\mathrm{O}_{2}} / Y_{\mathrm{O}_{2} f}\right)\left(\omega_{1 f}+\omega_{2 f}\right), \\
L_{\mathrm{H}_{2}}\left(s y_{\mathrm{H}_{2}}\right)-L_{\mathrm{O}_{2}}\left(2 y_{\mathrm{O}_{2}}\right)+L_{\mathrm{H}}\left(y_{\mathrm{H}}\right)=0
\end{gathered}
$$

and

$$
L(\theta)-q_{1} L_{\mathrm{H}_{2}}\left(s y_{\mathrm{H}_{2}}\right)+q_{2} L_{\mathrm{O}_{2}}\left(3 y_{\mathrm{O}_{2}}\right)=0,
$$

where the normalized reactant mass fractions $y_{\mathrm{H}_{2}}=Y_{\mathrm{H}_{2}} / Y_{\mathrm{H}_{2} f}$ and $y_{\mathrm{O}_{2}}=Y_{\mathrm{O}_{2}} / Y_{\mathrm{O}_{20}}$ are referred to their feed-stream values $Y_{\mathrm{H}_{2} f}$ and $Y_{\mathrm{O}_{20}}$, while the $\mathrm{H}$-atom mass fraction is normalized according to $y_{\mathrm{H}}=s Y_{\mathrm{H}} / Y_{\mathrm{H}_{2} f}$. The notation in Equations 6-8 has been shortened by introduction of the differential operators $L_{i}()=\rho D() / D t-\nabla \cdot\left[\mu \nabla() / S_{i}\right]$ and $L()=\rho D() / D t-\nabla$. $[\mu \nabla() / P r]$. Here, $s=\left(W_{\mathrm{O}_{2}} / W_{\mathrm{H}_{2}}\right)\left(Y_{\mathrm{H}_{2} S} / Y_{\mathrm{O}_{20}}\right)$ is an appropriate fuel-to-air mole ratio corresponding to the feed streams and $q_{1}=2\left(Y_{\mathrm{O}_{2} \mathrm{o}} / W_{\mathrm{O}_{2}}\right)$ $\left[\left(h_{\mathrm{H}}^{0} W_{\mathrm{H}}\right) /\left(c_{p} T_{\mathrm{rec}}\right)\right]$ and $q_{2}=(2 / 3)\left(Y_{\mathrm{O}_{2} /} / W_{\mathrm{O}_{2}}\right)\left[\left(2 h_{\mathrm{H}}^{0} W_{\mathrm{H}}-h_{\mathrm{H}_{2} \mathrm{O}}^{0} W_{\mathrm{H} \mathrm{I}_{2} \mathrm{O}}\right) /\left(c_{p} T_{\mathrm{ret}}\right)\right]$ are nondimensional enthalpies of formation. Integration of Equations 6-8, supplemented by the Burke-Schumann condition

$$
y_{\mathrm{O}_{2}} y_{\mathrm{H}_{2}}=0
$$

associated with the infinite rate of step $\mathbf{I}$, provides the reactant and $\mathrm{H}$-atom concentration and the temperature evolution everywhere in the flow, whereas the water-vapor concentration is obtained from the uncoupled equation

$$
L_{\mathrm{H}_{2} \mathrm{O}}\left(y_{\mathrm{H}_{2} \mathrm{O}}\right)+L_{\mathrm{O}_{2}}\left(y_{\mathrm{O}_{2}}\right)=0 \text {, }
$$

where $y_{\mathrm{H}_{2} \mathrm{O}}=(8 / 9)\left(Y_{\mathrm{H}_{2} \mathrm{O}} / Y_{\mathrm{O}_{2} \mathrm{O}}\right)$. 
Equation 6 follows from reaction I, clearly indicating that the rate of change of the quantity $s y_{\mathrm{H}_{2}}-3 y_{\mathrm{O}_{2}}$ is that of radical recombination. Integrating this equation across the radical-production layer gives the relation $s \nabla\left(y_{\mathrm{H}_{2}}\right)_{f} / S_{\mathrm{H}_{2}}=-3 \nabla\left(y_{\mathrm{O}_{2}}\right)_{0} / S_{\mathrm{O}_{2}}$ between the gradients of reactant mass fractions, indicating that the reactants are supplied by diffusion in to the radicalproduction surface according to the stoichiometry of the global reaction I, as explained above. Similarly, Equations 7 and 8 can be integrated to obtain expressions for the jumps in the slopes of the $\mathrm{H}$-atom and temperature profiles across the discontinuity surface. It is also worth remarking that, since the enthalpies of formation of water vapor and $\mathrm{H}$ atoms satisfy $h_{\mathrm{H}_{2} \mathrm{O}}^{o} W_{\mathrm{H}_{2} \mathrm{O}}+h_{\mathrm{H}}^{o} W_{\mathrm{H}} \simeq 0$, the heat release associated with the chain-branching step I is very small, and temperature variations are primarily associated with radical recombinations, thereby causing the jumps in the temperature gradient across the radical-production surface to be negligibly small. To further illustrate this, one can employ the above relation between $h_{\mathrm{H}_{2} \mathrm{O}}^{o}$ and $h_{\mathrm{H}}^{0}$ to show that the nondimensional parameters $q_{1}$ and $q_{2}$ defined before Equation 9 are approximately equal. Introducing now $q_{1}=q_{2}$ into Equation 8 and comparing the resulting equation with Equation 6 clearly exhibits the dependence of temperature on the radical-recombination rate.

Although it is in principle possible to solve the problem as formulated in Equations $6-10$, the integration constitutes a challenging free-boundary problem in which the location of the radical-production layer, where the slopes of the different species concentrations exhibit discontinuities, must be determined as part of the calculation. The solution can be faciliated by introducing the functions

$$
\begin{array}{ll}
X_{1}=s y_{\mathrm{H}_{2}}-3 y_{\mathrm{O}_{2}}, & \tilde{X}_{1}=s y_{\mathrm{H}_{2}} / S_{\mathrm{H}_{2}}-3 y_{\mathrm{O}_{2}} / S_{\mathrm{O}_{2}}, \\
X_{2}=s y_{\mathrm{H}_{2}}-2 y_{\mathrm{O}_{2}}+y_{\mathrm{H}}, & \tilde{X}_{2}=s y_{\mathrm{H}_{2}} / S_{\mathrm{H}_{2}}-2 y_{\mathrm{O}_{2}} / S_{\mathrm{O}_{2}}+y_{\mathrm{H}} / S_{\mathrm{H}}, \\
X_{3}=y_{\mathrm{H}_{2} \mathrm{O}}+y_{\mathrm{O}_{2}}, & \tilde{X}_{3}=y_{\mathrm{H}_{2} \mathrm{O}} / S_{\mathrm{H}_{2} \mathrm{O}}+y_{\mathrm{O}_{2}} / S_{\mathrm{O}_{2}}, \\
X_{4}=\theta-s q_{1} y_{\mathrm{H}_{2}}+3 q_{2} y_{\mathrm{O}_{2}}, & \widetilde{X}_{4}=\theta / P r-s q_{1} y_{\mathrm{H}_{2}} / S_{\mathrm{H}_{2}}+3 q_{2} y_{\mathrm{O}_{2}} / S_{\mathrm{O}_{2}}
\end{array}
$$

into Equations $6-8$ and 10 , giving the associated conservation equations

$$
\rho \frac{D X_{1}}{D t}-\nabla \cdot\left(\mu \nabla \tilde{X}_{1}\right)=\left(W_{\mathrm{O}_{2}} / Y_{\mathrm{O}_{2 f}}\right)\left(\omega_{1 f}+\omega_{2 f}\right)
$$


and

$$
\rho \frac{D X_{\alpha}}{D t}-\nabla \cdot\left(\mu \nabla \tilde{X}_{z}\right)=0, \quad \alpha=2,3,4
$$

Here, $\widetilde{X}_{1}$ and $\tilde{X}_{\alpha}$ are conserved scalars for the radical-producing reaction, while the gradients of $X_{1}$ and $X_{\alpha}$ have jumps across the thin radical-production layer, where both $\widetilde{X}_{1}$ and $X_{1}$ are zero. Fortunately, in the BurkeSchumann limit $y_{\mathrm{O}_{2}} y_{\mathrm{H}_{2}}=0$ of infinitely fast rate of the radical-production step $I, X_{1}$ can be obtained as a piecewise linear continuous function of $\widetilde{X}_{1}$, and we can similarly write $X_{2}, X_{3}$ and $X_{4}$ as well as the right-hand side of Equation 12 as piecewise continuous functions of $\tilde{X}_{1}, \tilde{X}_{2}, \widetilde{X}_{3}$ and $\tilde{X}_{4}$.

In the following, we replace in the formulation the scalars $\tilde{X}$ by their normalized form, given by the two modified mixture fractions

$$
\tilde{Z}_{1}=\frac{\tilde{s}_{1} y_{\mathrm{H}_{2}}+1-y_{\mathrm{O}_{2}}}{\tilde{s}_{1}+1}
$$

and

$$
\tilde{Z}_{2}=\frac{\tilde{s}_{2} y_{\mathrm{H}_{2}}+S_{\mathrm{O}_{2}} y_{\mathrm{H}} /\left(2 S_{\mathrm{H}}\right)+1-y_{\mathrm{O}_{2}}}{\tilde{s}_{2}+1},
$$

together with the modified reduced temperature

$$
\widetilde{\Theta}=\frac{S_{\mathrm{O}_{2}}\left(\theta-\theta_{o}\right) /(3 P r)-q_{1} \tilde{s}_{1} y_{\mathrm{H}_{2}}+q_{2}\left(y_{\mathrm{O}_{2}}-1\right)}{S_{\mathrm{O}_{2}}\left(\theta_{f}-\theta_{o}\right) /(3 P r)-q_{1} \tilde{s}_{1}-q_{2}}
$$

and the modified (potential) water-vapor mass fraction

$$
\tilde{Z}_{3}=1-y_{\mathrm{O}_{2}}-\frac{S_{\mathrm{O}_{2}}}{S_{\mathrm{H}_{2} \mathrm{O}}} y_{\mathrm{H}_{2} \mathrm{O}}
$$

where $\tilde{s}_{1}=(s / 3)\left(S_{\mathrm{O}_{2}} / S_{\mathrm{H}_{2}}\right)$ and $\tilde{s}_{2}=(s / 2)\left(S_{\mathrm{O}_{2}} / S_{\mathrm{H}_{2}}\right)$ are modified fuel-to-air stoichiometric mass ratios corresponding, respectively, to the overall reactions $3 \mathrm{H}_{2}+\mathrm{O}_{2} \rightarrow 2 \mathrm{H}_{2} \mathrm{O}+2 \mathrm{H}$ and $2 \mathrm{H}_{2}+\mathrm{O}_{2} \rightarrow 2 \mathrm{H}_{2} \mathrm{O}$, while $\theta_{o}=T_{o} / T_{\text {ref }}$ and $\theta_{f}=T_{f} / T_{\text {ref }}$ are the values of the nondimensional temperature in the oxidizer and fuel streams. These normalized coupling functions take the value $\tilde{Z}_{1}=\widetilde{Z}_{2}=\Theta=\widetilde{Z}_{3}=0$ in the oxidizer stream and $\tilde{Z}_{1}=\widetilde{Z}_{2}=\widetilde{\Theta}=\widetilde{Z}_{3}=1$ in 
the fuel stream. Introducing these variables reduces the problem to that of integrating the system

$$
\begin{aligned}
& \frac{D Z_{1}}{D t}-\frac{1}{\rho} \nabla \cdot\left(\frac{\mu}{S_{m 1}} \nabla \tilde{Z}_{1}\right)=\frac{1}{t_{1 f}} \Omega\left(\theta, y_{O_{2}}, y_{H}\right) \\
& \frac{D Z_{2}}{D t}-\frac{1}{\rho} \nabla \cdot\left(\frac{\mu}{S_{m 2}} \nabla \tilde{Z}_{2}\right)=0 \\
& \frac{D \Theta}{D t}-\frac{1}{\rho} \nabla \cdot\left(\frac{\mu}{S_{m 3}} \nabla \tilde{\Theta}\right)=0
\end{aligned}
$$

and

$$
\frac{D Z_{3}}{D t}-\frac{1}{\rho} \nabla \cdot\left(\frac{\mu}{S_{\mathrm{O}_{2}}} \nabla \widetilde{Z}_{3}\right)=0
$$

The quantity $t_{1 f}^{-1}=[2 /(s+3)]\left(k_{1 f} C_{M} \rho Y_{\mathrm{O}_{2} \alpha} / W_{\mathrm{O}_{2}}\right)$ that appears in Equation 18 is the inverse of the characteristic time of radical recombination corresponding to the elementary step $1 f$ evaluated at the reference temperature, with $C_{M}$ being the total number of moles per unit volume. In this formulation,

$$
\Omega\left(\theta, y_{\mathrm{O}_{2}}, y_{\mathrm{H}}\right)=\theta^{\mathrm{n}_{1} f^{-2}} y_{\mathrm{O}_{2}} y_{\mathrm{HI}}+\gamma \theta^{n_{2} f^{-2}} y_{\mathrm{H}}^{2}
$$

is the normalized recombination rate, and $S_{m 1}=\left(s_{1}+1\right) S_{\mathrm{O}_{2}} /\left(\tilde{s}_{1}+1\right)$, $S_{m 2}=\left(s_{2}+1\right) S_{\mathrm{O}_{2}} /\left(\tilde{s}_{2}+1\right)$ and $S_{m 3}=\left[\left(\theta_{\Gamma}-\theta_{o}\right) / 3-q_{1} s_{1}-q_{2}\right] S_{\mathrm{O}_{2}} /\left[S_{\mathrm{O}_{2}}\left(\theta_{f}\right.\right.$ $\left.\left.-\theta_{0}\right) /(3 P r)-q_{1} \tilde{s}_{1}-q_{2}\right]$ are mean transport numbers, with $s_{1}$ and $s_{2}$ denoting the stoichiometric fuel-air ratios $s_{1}=s / 3$ and $s_{2}=s / 2$. The parameter $y=2 k_{2 f \circ} / k_{1 f \infty}$ is twice the ratio of the rate constant of the elementary step $2 f$ to that of the step $1 f$, a quantity only weakly dependent on temperature. While the functions $\widetilde{Z}_{1}, \widetilde{Z}_{2}$, $\Theta$ and $\widetilde{Z}_{3}$ are conserved scalars in the thin radical-production layer, the coupling functions

$$
\begin{aligned}
& Z_{1}=\frac{s_{1} y_{\mathrm{H}_{2}}+1-y_{\mathrm{O}_{2}}}{s_{1}+1} \\
& Z_{2}=\frac{s_{2} y_{\mathrm{H}_{2}}+y_{\mathrm{H}} / 2+1-y_{\mathrm{O}_{2}}}{s_{2}+1}
\end{aligned}
$$




$$
\Theta=\frac{\left(\theta-\theta_{0}\right) / 3-q_{1} s_{1} y_{\mathrm{H}_{2}}+q_{2}\left(y_{\mathrm{O}_{2}}-1\right)}{\left(\theta_{f}-\theta_{o}\right) / 3-q_{1} s_{1}-q_{2}},
$$

and

$$
Z_{3}=1-y_{\mathrm{O}_{2}}-y_{\mathrm{H}_{2} \mathrm{O}}
$$

appearing in the Lagrangian derivatives of Equations 18-21 are not.

If we take into account the Burke-Schumann condition $y_{\mathrm{H}_{2}} y_{\mathrm{O}_{2}}=0$ for the fast, radical-producing shuffle reactions, then the scalars $Z_{1}, Z_{2}, \Theta$ and $Z_{3}$ can be expressed as piecewise linear, continuous functions of $\bar{Z}_{1}, \widetilde{Z}_{2}, \widetilde{\Theta}$ and $\tilde{Z}_{3}$, which are continuous functions with continuous derivatives at the thin radical-production sheet, where $\tilde{Z}_{1}$ takes its stoichiometric value $\tilde{Z}_{1 s}=1$ / $\left(\tilde{s}_{1}+1\right)$. These expressions are given by

$$
\begin{gathered}
y_{\mathrm{H}_{2}}=\left[\left(\tilde{s}_{1}+1\right) \tilde{Z}_{1}-1\right] / \tilde{s}_{1}=\left[\left(s_{1}+1\right) Z_{1}-1\right] / s_{1} \\
y_{\mathrm{H}}=\left(2 S_{\mathrm{H}} / S_{\mathrm{O}_{2}}\right)\left\{\left(\tilde{s}_{2}+1\right) \tilde{Z}_{2}-1-\tilde{s}_{2}\left[\left(\tilde{s}_{1}+1\right) \tilde{Z}_{1}-1\right] / \tilde{s}_{1}\right\} \\
=2\left\{\left(s_{2}+1\right) Z_{2}-1-s_{2}\left[\left(s_{1}+1\right) Z_{1}-1\right] / s_{1}\right\} \\
\theta=\theta_{o}+\left(3 \operatorname{Pr} / S_{\mathrm{O}_{2}}\right)\left\{\Theta\left[S_{\mathrm{O}_{2}}\left(\theta_{f}-\theta_{o}\right) /(3 P r)-q_{1} \tilde{s}_{1}-q_{2}\right]+q_{1}\left[\left(\tilde{s}_{1}+1\right) \tilde{Z}_{1}-1\right]+q_{2}\right\} \\
=\theta_{o}+3\left\{\Theta\left[\left(\theta_{f}-\theta_{o}\right) / 3-q_{1} s_{1}-q_{2}\right]+q_{1}\left[\left(s_{1}+1\right) Z_{1}-1\right]+q_{2}\right\}
\end{gathered}
$$

and

$$
y_{\mathrm{H}_{2} \mathrm{O}}=\left(S_{\mathrm{H}_{2} \mathrm{O}} / S_{\mathrm{O}_{2}}\right)\left(1-\widetilde{Z}_{3}\right)=\left(1-Z_{3}\right)
$$

for $\widetilde{Z}_{1}>\widetilde{Z}_{1 s}\left(y_{\mathrm{O}_{2}}=0\right)$ and

$$
\begin{gathered}
y_{\mathrm{O}_{2}}=1-\left(\tilde{s}_{1}+1\right) \widetilde{Z}_{1}=1-\left(s_{1}+1\right) Z_{1}, \\
y_{\mathrm{H}}=\left(2 S_{\mathrm{H}} / S_{\mathrm{O}_{2}}\right)\left[\left(\tilde{s}_{2}+1\right) \tilde{Z}_{2}-\left(\tilde{s}_{1}+1\right) \tilde{Z}_{1}\right]=2\left[\left(s_{2}+1\right) Z_{2}-\left(s_{1}+1\right) Z_{1}\right], \\
\theta=\theta_{o}+\left(3 \mathrm{Pr} / S_{\mathrm{O}_{2}}\right)\left\{\tilde{\Theta}\left[S_{\mathrm{O}_{2}}\left(\theta_{f}-\theta_{o}\right) /(3 P r)-q_{1} \tilde{s}_{1}-q_{2}\right]+q_{2}\left[\left(\tilde{s}_{1}+1\right) \tilde{Z}_{1}\right\}\right. \\
=\theta_{o}+3\left\{\Theta\left[\left(\theta_{f}-\theta_{o}\right) / 3-q_{1} s_{1}-q_{2}\right]+q_{2}\left[\left(s_{1}+1\right) Z_{1}\right\}\right.
\end{gathered}
$$


and

$$
y_{\mathrm{H}_{2} \mathrm{O}}=\left(\tilde{s}_{\mathrm{H}_{2} \mathrm{O}} / S_{\mathrm{O}_{2}}\right)\left[-\tilde{Z}_{3}+\left(\tilde{s}_{1}+1\right) \tilde{Z}_{1}\right]=\left[-\tilde{Z}_{3}+\left(s_{1}+1\right) Z_{1}\right]
$$

for $\tilde{Z}_{1}<\tilde{Z}_{1 s}\left(y_{\mathrm{H}_{2}}=0\right)$. Integration of Equations $18-21$ by use of these formulas circumvents the need to track the free boundary, thereby simplifying the solution procedure.

\section{GENERAL DEPENDENCES ON BOUNDARY TEMPERATURES AND STRAIN CONDITIONS}

Equations 18-21, supplemented with the continuity and momentum equations and with the expressions given in Equations 22 and 27-34, can be integrated with the previously stated boundary conditions in the feed streams to determine the evolution of the flow field. To perform the integration, one must in general supply initial conditions at $t=0$ as well as additional conditions at the boundaries of given system, which depend in general on the geometrical configuration considered. The character of the solution depends strongly on the value of the characteristic flowfield or strain time $t_{\mathrm{S}}$, an estimate of which being given for instance by the inverse of the magnitude of the local velocity gradient. Since heat release is associated with radical recombinations, existence of a flame requires that the strain conditions are such that $t_{\mathrm{S}} \gtrless t_{\mathrm{R}}$, where $t_{\mathrm{R}} \sim t_{1 f}$ is the characteristic time of the three-body recombination reactions. Under that condition, Equations $18-21$ represent satisfactorily the flowfield structure, leading to a description in which the fuel and oxidizer regions are separated by thin radical-production surfaces, and radicals spread throughout the flowfield, with heat release through radical recombinations proceeding in a distributed manner. As previously pointed out, the reactants can coexist with characteristic mass fractions of order $K_{\mathrm{H}}^{-1 / 2}$ within the radical-production layers, whose characteristic thickness is of order $\left(t_{\mathrm{S}} \mu / \rho\right)^{1 / 2} K_{\mathrm{H}}^{-1 / 2}$. As can be seen, the scalings associated with this thin layer, whose inner structure is investigated elsewhere (Sánchez et al., 1995), depend on the degree of sharpenning of the shuffle-reaction equilibrium through the parameter $K_{\mathrm{H}}$.

\section{The Effect of Low Boundary Temperatures}

The distinct Burke-Schumann structure presented here follows from the partial-equilibrium condition of the shuffle reactions and, consequently, is 
only strictly valid when the temperature is larger than the crossover temperature, a condition that cannot be satisfied everywhere if the reactants are supplied at ambient temperature. In regions where the temperature falls below crossover the shuffle reactions become too slow to maintain partial equiiibrium and the Burke-Schumann condition $y_{\mathrm{O}_{2}} y_{\mathrm{H}_{2}}=0$ must in principle be relaxed. Since radical recombinations do not lead to oxygen formation, the failure of the partial equilibrium assumption does not affect significantly the composition on the fuel side of the flow field, which remains free from $\mathrm{O}_{2}$. On the oxidizer side, however, modifications to the Burke-Schumann structure can be expected as the branching step I becomes slow below crossover. The fuel generated by radical recombination through the overall reaction II is no longer consumed at an infinite rate by step I, thereby causing the $\mathrm{H}_{2}$ steady state to break down. Nonnegligible amounts of molecular hydrogen may then appear on the oxidizer side, a result that can be observed, for instance, in numerical calculations of counterflows with detailed chemistry as the strain rate increases (DixonLewis and Missaghi, 1988). The amount of $\mathrm{H}_{2}$ generated depends primarily on the peak temperature achieved in the radical-production layer. In configurations with relatively high peak temperatures, the temperature decreases below crossover only far into the oxidizer region, where radicals exhibit small concentrations, and only a limited amount of molecular hydrogen can be produced. As the peak temperature approaches crossover, $\mathrm{H}_{2}$ non-steady-state effects are more pronounced, causing the description given by the reduced mechanism I-III to become increasingly inaccurate. Although quantitative departures from the Burke-Schumann structure postulated here thus emerge on the oxidizer side near extinction under most conditions, integration of Equations 18-21 nevertheless still gives a consistent description of the resultant flow field, provided that the peak temperature of the solution remains above crossover. The oxidizerside inaccuracy in the chemistry should, however, be kept in mind in applications.

\section{Extinction Criterion}

A simple extinction criterion readily follows from the previous considerations, namely, extinction occurrence is associated with strain conditions that reduce the peak temperature at the radical-production layer to the corssover temperature. If one employs the crossover temperature as the reference temperature, $T_{\text {rep }}$ in the definition of $\theta$, then extinction occurs where the peak value of the modified reduced temperature $\Xi$ decreases 
below a critical value given by

$$
\widetilde{\Theta}_{\mathrm{ext}}=\frac{S_{\mathrm{O}_{2}}\left(1-\theta_{0}\right) /(3 P r)-q_{2}}{S_{\mathrm{O}_{2}}\left(\theta_{f}-\theta_{0}\right) /(3 P r)-q_{1} \tilde{s}_{1}-q_{2}} .
$$

Note that, in the evaluation of the crossover temperature, it is necessary to take into account the high third-body efficiency of water vapor. Failure to do so would result in underpredictions of the crossover temperatures by as much as $200 \mathrm{~K}$, thereby also leading to significant underpredictions of the strain time $t_{\mathrm{s}}$ at extinction. Integration procedures for Equations 18-21 can append Equation 35 and test the inequality $\widetilde{\Theta}>\widetilde{\Theta}_{\mathrm{ext}}$ at $\widetilde{Z}_{\mathrm{t}}=1 /\left(\tilde{s}_{1}+1\right)$, to ascertain whether conditions approach extinction. It should be emphasized that this criterion is only approximate because the formulation breaks down as extinction is approached, but it does give correct general functional dependences and orders of magnitude.

\section{Weakly Strained Solutions}

Much information on the character of the solution can be gathered by momentarily neglecting unsteady variations, thereby simplifying the left-hand side of Equation 18 to a convective-diffusive operator proportional to the inverse of the strain time $t_{\mathrm{s}}^{-1}$. Comparing then the transport terms of this equation with the recombination term reveals that, in the limit of weakly strained flow fields, $t_{\mathrm{S}} \gg t_{\mathrm{R}}$, Equation 18 simplifies to $\Omega=0$, a condition that can only be satisfied if the $\mathrm{H}$-atom mass fraction vanishes, as can be seen from Equation 22. This limiting case, previously encountered by Sánchez et al. (1995), leads to a flow field in which five distinctive regions can be found.

The flow is divided into radical-free convective-diffusive oxidizer and fuel regions, where $y_{\mathrm{H}_{2}}=0$ and $y_{\mathrm{O}_{2}}=0$, respectively, separated by relatively thin reaction regions, which posses a three-layer inner structure, as explained below. The first-order solution to the radical-free regions can be obtained by integration of Equations 19-21, while Equation 18 simply reduces to $y_{\mathrm{H}}=0$. From the definition of the coupling function $\tilde{Z}_{2}$ given in Equation 15, it is clear that the structure of these regions is simply that of the Burke-Schumann solution corresponding to the overall reaction $2 \mathrm{H}_{2}+\mathrm{O}_{2} \rightarrow 2 \mathrm{H}_{2} \mathrm{O}$, with the location of the reaction region being given in the first approximation by the equation $\widetilde{Z}_{2}=1 /\left(\tilde{s}_{2}+1\right)$. For instance, integration of Equation 19 with $y_{\mathrm{H}}=0$ across the reaction surface yields $s \nabla\left(y_{\mathrm{H}_{2}}\right)_{f} / S_{\mathrm{H}_{2}}=-2 \nabla\left(y_{\mathrm{O}_{2}}\right)_{0} / S_{\mathrm{O}_{2}}$ as a relation between the diffusion velocities 
of the reactants into this layer, indicating that $\mathrm{O}_{2}$ and $\mathrm{H}_{2}$ are supplied in stoichiometric proportions according to the above overall reaction. The solution also provides the value of the peak temperature in the first approximation. If the reactant and thermal diffusivities were equal and the reactants were introduced at equal temperatures into the combustion system, with heat losses to walls neglected, then $\tilde{Z}_{2}=\widetilde{\Theta}$, and the value of the peak temperature would be the adiabatic flame temperature of the global reaction $2 \mathrm{H}_{2}+\mathrm{O}_{2} \rightarrow 2 \mathrm{H}_{2} \mathrm{O}$, nondimensionally

$$
\theta_{\text {peik }}=1+\frac{3 s}{s+2}\left(q_{2}-\frac{2}{3} q_{1}\right)
$$

where we have now selected the feed-stream temperature as the reference temperature in the definition of $\theta$. Differential-diffusion effects can be seen to increase significantly this value, primarily through the large diffusivity of the fuel, yielding maximum flame temperatures in counterflow configurations at low strain conditions about $300 \mathrm{~K}$ higher than the corresponding adiabatic flame temperatures (Sánchez et al, 1995).

The inner structure of the reaction surfaces is simply determined by a diffusion-recombination balance in Equation 18, while the values of the coupling functions $\tilde{Z}_{2}, \Theta$ and $\tilde{Z}_{3}$ across these thin surfaces remain constant and equal to the values obtained from the solution to the outer convectivediffusive regions (e,g., $\tilde{Z}_{2}=1 /\left(\tilde{s}_{2}+1\right)$ ). It can be shown that the characteristic thickness of these surfaces is of order $\left(t_{\mathrm{S}} \mu / \rho\right)^{1 / 2}\left(t_{\mathrm{R}} / t_{\mathrm{S}}\right)^{1 / 3}$, while the characteristic value of the small $\mathrm{H}$-atom mass fractions that appear inside is of order $\left(t_{\mathrm{S}} / t_{\mathrm{R}}\right)^{1 / 3}$. Because of the partial-equilibrium condition of the shuffe reactions, $y_{\mathrm{O}_{2}} y_{\mathrm{H}_{2}}=0$, and reactants can coexist only in thin radical-production surfaces, located between two thicker radical-recombination layers, giving a total of five different regions in the structure. This rather complex picture would be captured automatically by the numerical integration of Equations 18-21 when $t_{\mathrm{S}} \gg t_{\mathrm{R}}$.

As the value of the strain time increases, the thickness of the radicalrecombination surfaces decreases. Correspondingly, finite-rate effects become less significant, and the maximum temperature approaches the peak value corresponding to the solution of the outer convective-diffusive regions. As a result, the value of the parameter $K_{H}$ becomes smaller, giving a less pronounced partial equilibrium of the shuffle reactions, and causing the radicalproduction surface, initially thinner than the radical-recombination layers, to grow. At a critical value of $t_{\mathrm{S}}$, determined in the first approximation by the condition $\left(t_{\mathrm{R}} / t_{\mathrm{S}}\right)^{1 / 3}=K_{\mathrm{H}}^{-1 / 2}$, the radical-production and radical-recombina- 
tion layers become comparable in size, merging into a single reaction layer where reactants can coexist. The solution in this reaction layer is then obtained by replacing the Burke-Schumann condition $y_{\mathrm{O}_{2}} y_{\mathrm{H}_{2}}=0$ with the algebraic expressions given in Equations 1-3 (Sánchez et al., 1995).

\section{FLAME DEVELOPMENT IN THE LAMINAR MIXING LAYER}

\section{Mixing-Layer Structure}

The generalized Burke-Schumann formulation presented above is employed now to describe the process leading to flame formation in the hydrogen-air mixing layer for free-stream temperatures above the crossover temperature corresponding to the second explosion limit. The solution that emerges, which evolves continuously from an initially frozen flow to a fully developed diffusion flame, exhibits three distinct regions as the flow moves downstream, namely, an induction region, a thin transition layer and a recombination region. The structure of the resulting flow field is sketched in Figure 2, where the air-side free-stream velocity $u_{0}$ is chosen as the characteristic value of the velocity across the mixing layer.

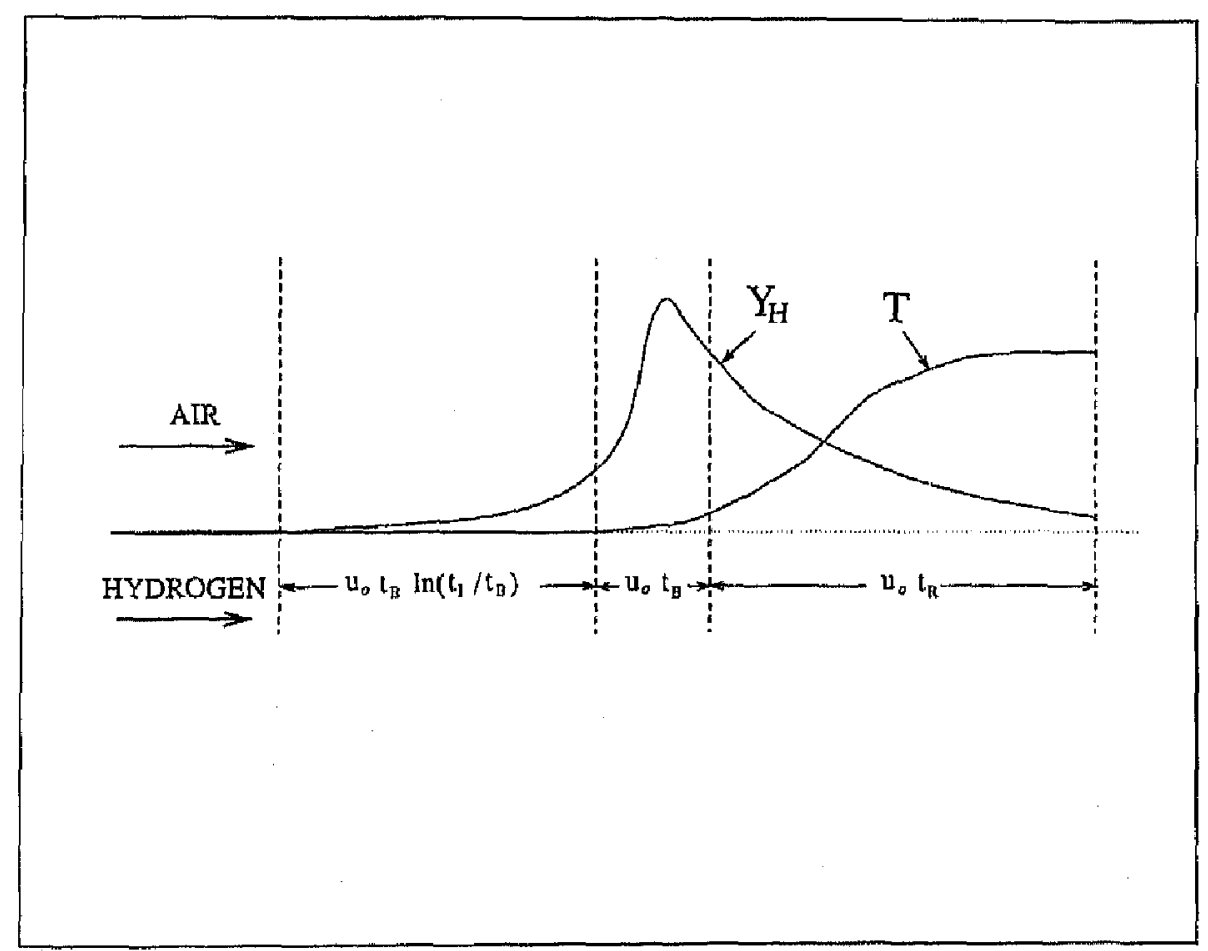

FIGURE 2 The asymptotic structure of the hydrogen-air mixing layer for free-stream temperatures above crossover. 
Description of the chemistry in the induction region requires consideration of initiation steps, such as $\mathrm{H}_{2}+\mathrm{O}_{2} \stackrel{9 g}{\rightarrow} \mathrm{HO}_{2}+\mathrm{H}$ and $\mathrm{H}_{2}+\mathrm{O}_{2} \stackrel{10 \rho}{\longrightarrow}$ $\mathrm{OH}+\mathrm{OH}$, together with branching reactions (the forward reactions 3-5). Over a wide range of temperatures typical of hydrogen-air combustion, these initiation reactions are extremely slow, with characteristic times $t_{1} \sim\left(k_{g f} C_{M}\right)^{-1}$ being much larger than that of the recombination reactions $t_{\mathrm{R}} \sim t_{1 f}$, which in turn is larger than that of the branching reactions $t_{\mathrm{B}} \sim\left(k_{3 f} C_{M}\right)^{-1}$ at temperatures above crossover. Therefore, reactions $9 f$ and $10 f$, which are necessary to ensure the early radical buildup from the initially forzen state, have a negligible effect once trace amounts of radicals are present, and they were appropriately neglected in deriving the reduced mechanism I-III.

The analysis of the induction region, which is presented elsewhere (Sánchez et al., 1997), reveals that, because of the autocatalytic character of radical branching, the concentration of radicals increases exponentially with downstream distance. For temperatures sufficiently above crossover, the time of radial recombination is much larger than that of branching, $t_{\mathrm{R}} \gg t_{\mathrm{B}}$, and only a very limited amount of heat is released in this branched-chain region, where radical concentrations grow without a significant temperature increment. This isothermal induction region extends over a distance of order $u_{\mathrm{R}} t_{\mathrm{B}} \ln \left(t_{\mathrm{T}} / t_{\mathrm{B}}\right)$, ending at a location where the radical mass fractions increase to values of order unity such that the rates of the reverse reactions $3-5$, as well as that of reaction 6 , become comparable to those of the branching steps, thereby ushering in a thin transition region, of characteristic thickness $u_{o} t_{\mathrm{B}}$, across which the shuffle reactions 3-6 reach partial equilibrium. Radical concentrations peak at this location and start decreasing downstream because of three-body recombination reactions in a region of large characteristic thickness $u_{\theta} t_{\mathrm{R}} \gg u_{o} t_{\mathrm{B}}$, where the temperature increases as the diffusion flame develops, a process that is described below by integration of the boundary-layer form of Equations 18-21.

\section{Conservation Equations}

In the formulation, $x$ and $y$ will be the coordinates in the streamwise and transverse directions, with $u$ and $v$ being their corresponding velocity components. The air and fuel streams occupy initially the upper $(y>0)$ and lower $(y<0)$ sides, merging at $x=0$ where mixing and reaction begin. As before, the subscripts $o$ and $f$ denote free-stream conditions on the air and fuel sides, respectively. Since in most practical applications the oxidizer-side temperature is larger than the fuel-side temperature, we choose the former 
as the relevant reference temperature $T_{\text {rof }}=T_{0}$. To simplify the formulation, it is convenient to introduce a transverse similarity coordinate $\eta=\left[u_{0} /\right.$ $\left.\left(2 \mu_{o} \rho_{n}\right)\right]^{1 / 2} \int_{0}^{n} \rho(x, \bar{y}) d \bar{y} / x^{1 / 2}$ and a rescaled streamwise coordinate $\xi=x /\left(\eta_{v} t_{1 f}\right)$ measured with the characteristic distance of radical recombination $u_{t} t_{1, f}$, together with a nondimensional stream function $F$, such that $u=u_{0} F^{\prime}$ and $\rho v=\left[\rho_{0} \mu_{0} u_{0} /(2 x)\right]^{1 / 2}\left(\eta F^{\prime}-F\right)$, where the prime denotes diflerentiation with respect to $\eta$. With these new variables, the momentum equation simplifies to

$$
F^{\prime \prime \prime}+F F^{\prime \prime}=0,
$$

while Equations $18-21$ reduce to

$$
\begin{aligned}
& F^{\prime} \frac{\partial Z_{1}}{\partial \xi}-\frac{F Z_{1}^{\prime}+\tilde{Z}_{1}^{\prime \prime} / S_{m 1}}{2 \xi}=\Omega\left(0, y_{O_{2}}, y_{H}\right), \\
& F^{\prime} \frac{\partial Z_{2}}{\partial \xi}-\frac{F Z_{2}^{\prime}+\tilde{Z}_{2}^{\prime \prime} / S_{m 2}}{2 \xi}=0 \\
& F^{\prime} \frac{\partial \Theta}{\partial \xi}-\frac{F \Theta^{\prime}+\tilde{\Theta}^{\prime \prime} / S_{m 3}}{2 \xi}=0
\end{aligned}
$$

and

$$
F^{\prime} \frac{\partial Z_{3}}{\partial \xi}-\frac{F Z_{3}^{\prime}+\tilde{Z}_{3}^{\prime \prime} / S_{\mathrm{O}_{2}}}{2 \xi}=0
$$

In deriving these equations we have assumed that the product $\rho \mu$ remains constant everywhere, a widely used simplification in analyses of boundarylayer flows (unity Chapman-Rubesin parameter). Although this assumption may introduce non-negligible inaccuracies in undiluted hydrogen-air systems because of the mean-molecular-weight dependence $\rho \mu \propto \bar{W}^{-3 / 2}$ (Sánchez et al., 1996), its adoption here facilitates considerably the flow description, leading to a selfsimilar solution for the stream function, $F$, which is determined by integration of the uncoupled Equation 37 with boundary conditions $F^{\prime}(\infty)=1, F^{\prime}(-\infty)=u_{f} / u_{o}$ and $F(0)=0$.

Equations $38-41$ must be integrated with boundary conditions $\widetilde{Z}_{1}=\widetilde{Z}_{2}=$ $\Theta=\widetilde{Z}_{3}=0$ at $\eta=\infty$ and $\widetilde{Z}_{1}=\widetilde{Z}_{2}=\Theta=\widetilde{Z}_{3}=1$ at $\eta=-\infty$, and with initial conditions at $\xi=\xi_{t}=x_{t} /\left(u_{o} t_{1 j}\right)$ given by the solution downstream from the transition layer, with $x_{t}$ being of order $u_{o} t_{\mathrm{B}} \ln \left(t_{1} / t_{\mathrm{B}}\right)$. Note that, because of the large value of $\ln \left(t_{\mathrm{I}} / t_{\mathrm{B}}\right)$, the value of $\xi_{t}$ is in general a quantity of order unity, becoming small only for free-stream temperatures far above crossover 
( 21500 at atmospheric conditions), for which conditions the branching reactions become sufficiently fast to give small values of $\xi_{t} \simeq t_{\mathrm{B}} \ln \left(t_{\mathrm{l}} / t_{\mathrm{B}}\right) / t_{\mathrm{R}}$.

\section{Transition Layer}

Because of the exponential radical growth of the branching process, the radical and water-vapor mass fractions evolve from exponentially small values to those corresponding to equilibrium of the shuffle reactions over a distance of order $u_{o} t_{B}$, while the reactant mass fractions vary from values of the order of their frozen concentrations to the values corresponding to equilibrium of the shuffle reactions across the same layer. Therefore, the characteristic streamwise thickness of the transition layer is a small quantity of order $t_{\mathrm{B}} / t_{\mathrm{R}}$ when expressed in the rescaled coordinate $\xi$. Introducing this scaling into Equations 38-41 indicates that convection is the dominant transport mechanism across this layer, where recombinations have no time to occur, thereby reducing the conservation equations to $\left(\partial Z_{1}\right)(\partial \xi)=\left(\partial Z_{2}\right) \quad(\partial \xi)=(\partial \Theta)(\partial \xi)=\left(\partial Z_{3}\right)(\partial \xi)=0$. These equations can be readily integrated to give

$$
\begin{gathered}
Z_{1 t}=\frac{s_{1} y_{\mathrm{H}_{2 \mathrm{~F}}}+1-y_{\mathrm{O}_{2 \mathrm{~F}}}}{s_{1}+1}, \\
Z_{2 t}=\frac{s_{2} y_{\mathrm{H}_{2 \mathrm{~F}}}+1-y_{\mathrm{O}_{2 \mathrm{~F}}}}{s_{2}+1} \\
\Theta_{t}=\frac{\left(\theta_{\mathrm{F}}-1\right) / 3-q_{1} s_{1} y_{\mathrm{H}_{2 \mathrm{~F}}}+q_{2}\left(y_{\mathrm{O}_{2 \mathrm{~F}}}-1\right)}{\left(\theta_{f}-1\right) / 3-q_{1} s_{1}-q_{2}}
\end{gathered}
$$

and

$$
Z_{3 t}=1-y_{\mathrm{O}_{2 F}},
$$

where the subscript $t$ denotes the profiles immediately downstream the transition region, and

$$
y_{\mathrm{H}_{2 \mathrm{~F}}}=1-\frac{\int_{-\infty}^{\eta} \exp \left[-S_{\mathrm{H}_{2}} \int_{0}^{\bar{\eta}} F(\tilde{\eta}) d \tilde{\eta}\right] d \bar{\eta}}{\int_{-\infty}^{\infty} \exp \left[-S_{\mathrm{H}_{2}} \int_{0}^{\tilde{\eta}} F(\tilde{\eta}) d \tilde{\eta}\right] d \bar{\eta}},
$$




$$
y_{\mathrm{O}_{2 \mathrm{~F}}}=\frac{\int_{-\infty}^{\eta} \exp \left[-S_{\mathrm{O}_{2}} \int_{0}^{\eta} F(\tilde{\eta}) d \tilde{\eta}\right] d \bar{\eta}}{\int_{-\infty}^{\infty} \exp \left[-S_{\mathrm{O}_{2}} \int_{0}^{n} F(\tilde{\eta}) d \tilde{\eta}\right] d \bar{\eta}}
$$

and

$$
\theta_{\mathrm{F}}=\theta_{f}+\left(1-\theta_{f}\right) \frac{\int_{-\infty}^{\eta} \exp \left[-\operatorname{Pr} \int_{0}^{\pi} F(\tilde{\eta}) d \tilde{\eta}\right] d \bar{\eta}}{\int_{-\infty}^{\infty} \exp \left[-\operatorname{Pr}_{r} \int_{0}^{\bar{\eta}} F(\tilde{\eta}) d \tilde{\eta}\right] d \bar{\eta}}
$$

are the selfsimilar profiles of reactants and temperature cotresponding to the frozen solution, obtained by integration of

$$
\begin{aligned}
& y_{\mathrm{H}_{2} \mathrm{~F}}^{\prime \prime}+S_{\mathrm{H}_{2}} F y_{\mathrm{H}_{2 \mathrm{~F}}}^{\prime}=0, \\
& y_{\mathrm{O}_{2 \mathrm{~F}}}^{\prime \prime}+S_{\mathrm{O}_{2}} F y_{\mathrm{O}_{2 \mathrm{~F}}}^{\prime}=0
\end{aligned}
$$

and

$$
\theta_{\mathrm{F}}^{\prime \prime}+\operatorname{PrF} \theta_{\mathrm{F}}^{\prime}=0
$$

with boundary conditions $y_{\mathrm{H}_{2 \mathrm{r}}}(\infty)=y_{\mathrm{O}_{2 \mathrm{~F}}}(\infty)-1=\theta_{\mathrm{F}}(\infty)-1=0$ and $y_{\mathrm{H}_{2} \mathrm{~F}}$ $(-\infty)-1=y_{\mathrm{O}_{2 F}}(-\infty)=\theta_{\mathrm{F}}(-\infty)-\theta_{f}=0$.

The initial temperature and species profiles, as well as the profiles $\tilde{Z}_{1 \text { t }}$, $\widetilde{Z}_{2 t}, \widetilde{\Theta}_{t}$ and $\widetilde{Z}_{3 t}$ to be employed as initial conditions for the integration of Equations $38-41$, can be easily obtained by combining Equations $42-45$ with the expressions given in Equations 27-34. The initial position of the radical-production layer, where the reactant mass fractions are zero, can be determined from Equation 42 as the value of $\eta$ at which $s_{1} y_{\mathrm{H}_{2} \mathrm{~F}}-y_{\mathrm{O}_{2 \mathrm{~F}}}$ vanishes. The resultant transverse location generally lies within the oxidizer stream, partly because of the large values of $s_{1}=s / 3$ typical of hydrogen-air nonpremixed combustion and partly because of the high diffusivity of hydrogen. The temperature increase from the forzen solution and the H-atom and water-vapor mass fractions all peak at this location, with maximum values that can be computed easily from Equations 43-45. In particular, since radical recombinations have a negligible effect in this thin transition layer, the temperature increments are very limited, being identically zero if 
the heat release associated with the global branching step I is entirely neglected, as can be seen from Equation 44 with $q_{1}=q_{2}$. It is also worth remarking that, in the limit of equal reactant and thermal diffusivities, the peak temperature immediately downstream from the transition region in the initially isothermal mixing layer reduces to

$$
\theta_{t_{\max }}=1+\frac{3 s}{s+3}\left(q_{2}-q_{1}\right),
$$

which corresponds to the adiabatic flame temperature of the global reaction $3 \mathrm{H}_{2}+\mathrm{O}_{2} \stackrel{\mathrm{I}}{\rightarrow} 2 \mathrm{H}_{2} \mathrm{O}+2 \mathrm{H}$.

\section{Numerical Results}

Radical and temperature profiles corresponding to the isovelocity, initially isothermal mixing layer obtained by integration of Equations 38-40 supplemented by Equations 27-29 and 31 33 are exhibited in Figure 3 for different values of $\xi$. In the calculations, initialized at $\xi_{t}=1$, an air-to fuel-mass ratio $s=10$ is employed and the transport numbers are taken to be $S_{\mathrm{H}_{2}}=0.19, S_{\mathrm{O}_{2}}=0.74, S_{\mathrm{F}}=0.12$ and $P r=0.74$, while we have chosen $q_{1}=2$ and $q_{2}=2$ as representative values for the heat-release parameters. In making this choice we implicitly neglect the small heat release associated with step I. The integrations are performed with $\gamma=0.7$, a value selected from evaluations of the reaction-rate constants $k_{1 f}$ and $k_{2 f}$ at high temperature with third-body effectiveness taken into account.

The results of the computation indicate that the solution undergoes a very rapid initial evolution, a behavior that would also emerge in the absence of radical recombinations, i.e., if the chemistry term was set equal to zero in the numerical integration. To understand this effect, one must recall that in the transition layer the effect of transverse diffusion was negligible in determining $\tilde{Z}_{1 v}, \tilde{Z}_{2 v}, \widetilde{\Theta}_{t}$ and $\tilde{Z}_{3 t}$. Hence, these initial profiles are not selfsimilar, e.g., $F Z_{1 t}^{\prime}+\widetilde{Z}_{1 t}^{\prime \prime} / S_{m 1} \neq 0$, and they must evolve to accommodate for the different species diffusivities.

Observation of Figure 3 also reveals that, although radicals are steadily consumed as the flow moves downstream, the consumption rate decreases significantly as the $\mathrm{H}$-atom mass fraction becomes smaller. This decrease is partially attributable to radical depletion itself, through the radical-concentration dependence of the reaction rates, and also partially attributable to the algebraic temperature dependence of the reaction-rate constants, which 

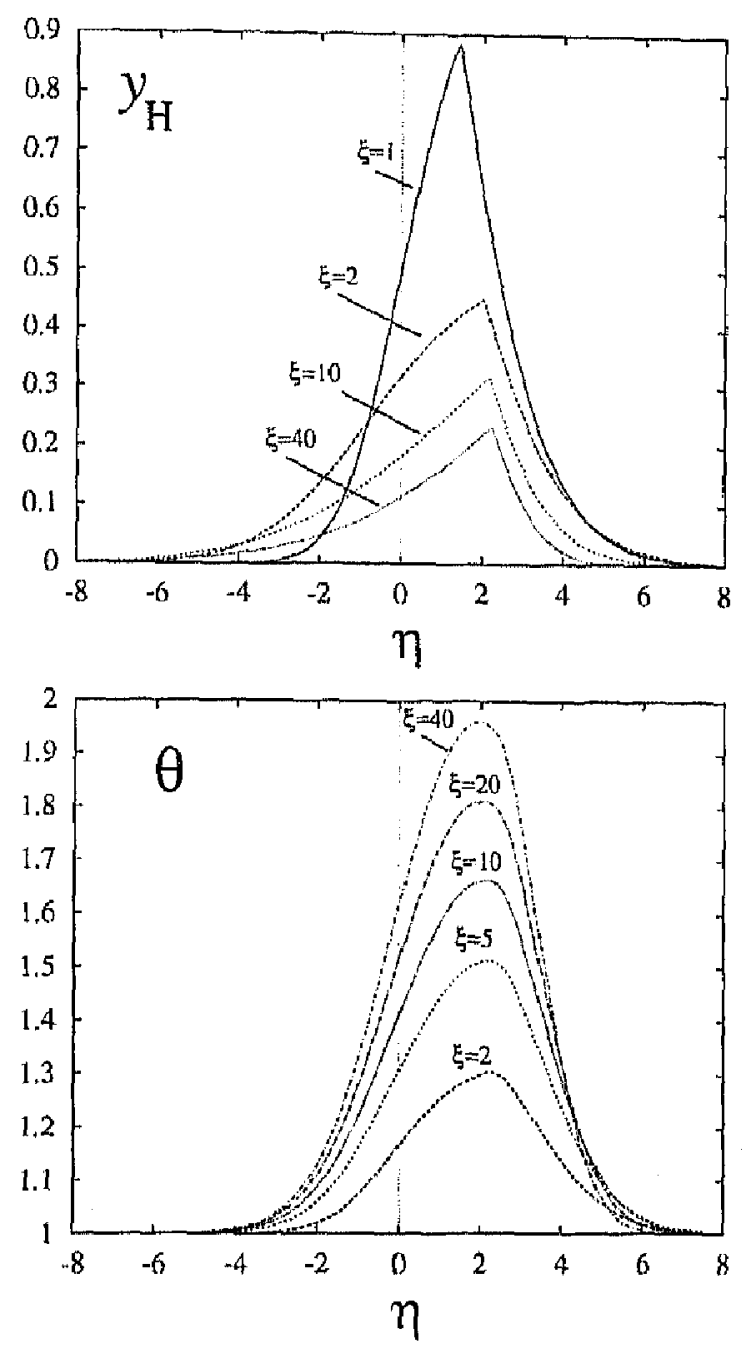

FIGURE 3 H-atom and temperature profiles as obtained by integration of Equations 38-40 with $s=10, S_{\mathrm{H}_{2}}=0.19, S_{\mathrm{O} 2}=0.74, S_{\mathrm{H}}=0.12, P r=0.74, q_{1}=2$ and $q_{2}=2$.

decreases the rates with increasing temperature. Far downstream the solution approaches the five-layer asymptotic structure corresponding to the case $t_{\mathrm{S}} \gg t_{\mathrm{R}}$ previously discussed, with a radical-recombination layer of characteristic thickness $\xi^{-1 / 3}$ when measured with the similarity coordinate $\eta$. Since the $\mathrm{H}$ atoms are very diffusive, radicals are seen to spread across the mixing layer even for relatively large values of $\xi$. The effect of radical diffusion is more significant on the fuel side of the mixing layer, where the recombination rate is restricted to that of reaction $2 f$, thereby yielding algebraicly decaying $\mathrm{H}$-atom profiles as $\eta \rightarrow-\infty$ (Sánchez et al., 1995).

As previously explained, because of the infinite rate of the global step I, the radical profiles exhibit discontinuties in their slopes at the radicalproduction layer, whose location varies as the flow moves downsteam. In 
the formulation employed, with generalized coupling functions utilized as integration variables, the position of this discontinuity layer does not have to be explicitly tracked in the calculation, considerably simplifying the numerical procedure. It is interesting to note that, since radical-branching heat release is neglected here, the resulting temperature profiles are smooth everywhere. A more accurate evaluation of $q_{1}$ and $q_{2}$ would slightly modify the solution; the transition to partial equilibrium across the transition region would not be isothermal, and the temperature profiles in the recombination region would have small slope discontinuities at the radical-production layer, a characteristic that can be observed in the results of other asymptotic analyses that take into account the heat release associated with radical production through step I (Sánchez et all, 1995).

\section{CONCLUSIONS}

The main general result of the present study is embodied in the generalized Burke-Schumann conservation equations given in Equations 18-21. These equations exhibit a reaction sheet at which the initial reactants are consumed, but the equations are expressed in a form that makes it unnecessary to track the sheet in performing numerical computations. The subsidiary algebraic relationships given in Equations 27-34 effectively track the sheet automatically, in addition accounting for different diffusion coefficients for all chemical species and for heat. The stoichiometry of this new reaction sheet is such that three hydrogen molecules are consumed, instead of two, for each oxygen molecule consumed. The additional hydrogen molecule generates two hydrogen atoms at the sheet, which recombine in a distributed manner throughout the Burke-Schumann diffusion zones, at a rate given by Equation 22, which appears as the source term in Equation 18. The analysis thus generalizes the Burke-Schumann description not only by allowing for differing diffusivities but also by accounting for distributed recombination and the associated distributed heat release, which dominates the chemical energy liberation in the flow.

The new model provides a formulation that is well adapted to numerical treatment because it eliminates all disparate chemical time scales from the problem, removing any stiffness. The recombination time is the characteristic chemical time for the computation. This simplified formulation is estimated to produce reasonable accuracy in describing hydrogen-oxygen diffusion flames, with nitrogen dilution, over about three orders of magnitude in a characterisic flow time, such as the reciprocal of a strain rate, 
whenever conditions of ambient pressure, feed-stream temperature and dilution lead to adiabatic flame temperatures between about $1000 \mathrm{~K}$ and $3000 \mathrm{~K}$; the low-temperature limit increases gradually with increasing pressure, reaching about $1500 \mathrm{~K}$ at $50 \mathrm{~atm}$, as determined by estimates of the crossover temperature. The formulation thus is accurate over a wide range of conditions of practical interest.

Use of the formulation was illustrated by application to a flame in a planar laminar mixing layer between parallel fuel and oxidizer streams. The present methodology can, however, be applied to many other configurations. Two of the simplest are the stagnant mixing layer and the counterflow diffusion flame; results of the formulation would exhibit, for example, the one-third-power dependence of the maximum radical concentration on the strain rate, found by Lee and Chung (1994) for the stagnant mixing layer at large residence times and also by Sánchez et al. (1995) for the counterflow flame when the reciprocal of the strain rate is large compared with the recombination time, giving a five-layer structure. Another configuration is the diffusion flame anchored in the recirculating wake of a blunt splitter plate, investigated by Higuera and Liñán (1996) for the ordinary Burke-Schumann limit of infinite Damköhler number; for this problem the present formulation would enable influences of finite-rate effects to be clarified, determining, for example, conditions for diffusionflame liftoff to occur, through application of the extinction criterion given in Equation 35. Combustion of a liquid oxygen dorplet in a gaseous hydrogen atmosphere could also be studied by the present formulation. Since the analysis results in predictions of radical concentrations, for example, $\mathrm{O}$-atom concentrations from Equation 3, it can also be employed in studying NO production in hydrogen-oxygen diffusion flames. Addition of slow chemistry of this kind for trace species becomes straightforward by appending appropriate further equations and rates to Equations 18-21.

One of the most attractive applications of the present procedure, however, is in direct numerical simulations of turbulent flows. It has proven difficult to include full hydrogen-oxygen chemistry in such simulations because of the widely differing time scales of chain-carrying and recombination steps. The generalized Burke-Schumann formulation overcomes these disparate-time complications, facilitating both computations and modeling of turbulent diffusion flames. It would be of interest to explore how much progress can be made in improving understanding of turbulent nonpremixied hydrogen-oxygen combustion through use of the present methodology. 
Balakrishtıan, G., Smooke, M. D. and Williams, F. A. (1905) A numerical investigation of extinction and ignition limits in laminar nonpremixed connterflowing hydrogen air streams for both elementary and reduced chemistry. Combust. Flame, 102, 329.

Balakrishnat, G. and Williams, F.A. (1994) Turbulent combustion regimes for hypersonic flows employing hydrogen-iar diflusion flames. J. Prop. Power. 10, 434.

Bilger, R. W. (1989) Turbulent diffusion flames. Anmal Review of Fluid Mechanics, 21, 101.

Burke, S. P. and Schumann, T. E. W. (1928) Diffusion flames. Ind-Eng. Chem., 20, 998.

Dixon-Lewis, G. and Missaghi, M. (1988) Structure and extinction limits of counterflow diffusion flames of hydrogen-nitrogen mixtures in air. In Twenty-Second Symposium (International) on Combustion. The Combustion Institute, Pilciburgh, PA, pp. 1461-1470.

Figueira Da Silva, L. F., Deshates, B. and Champion, M. (1993) Some specific aspects of combustion in supersonic $\mathrm{H}_{2}$-air laminar mixing layers. Comb. Sci. and Tech, 89, 317.

Gutheil, E., Balakrishnan, G. and Williams, F. A. (1993) Structurc and extinction of hydrogenait diffusion flames. In Peters, N. and Rogg, B. (Eds.) Reduced Kinetic Mechanisms for Applications in Combustion Systems, Springer Verlag, Germany. Chap. 11, pp. 177-195.

Gutheil, E. and Williams, F. A. (1990) A numerical and asymptotic investigation of structures of hydrogen-air diffusion flames at pressures and temperatures of high-speed propulsion. In Twenty-Third Symposium (International) on Combustion, The Combustion Institute, Pittsburgh, PA, pp. 513-521.

Higuera, F. and Linán, A. (1996) Flow field of a diffusion flame attached to a thick-walled injector between two coflowing reactant streams. J. Fluid Mech., 329, 389.

Janicks, J, and Kollmann, W. (1979) A two-variables formalism for the treatment of chemical reactions in turbulent $\mathrm{H}_{2}$-air diflusion flames. In Seventeenth Sympositum (International) on Combustion. The Combustion Institute, Pittsburgh, PA, pp. $421 \sim 430$.

$\mathrm{Ju}, \mathrm{Y}$. and Niioka, T. (1994) Reduced kinetic mechanism of ignition for nonpremixed hydrogen/air in a supersonic mixing layer. Combust. Flane, 99, 240.

Kreutz, T. G. and Law, C. K. (1996) Ignition in nonpremixed counterflowing hydrogen versus heated air: computational study with detailed chemistry. Combust. Flame, 104, 157.

Lee, S. R. and Chung, S. H. (1994) On the structure of hydrogen diffusion fames with reduced kinetic mechanisms. Combust. Sci, Tech, 96, 247.

Lewis, B. and Von Elbe, G. (1987) Combustion, Flames and Explosions of Gases. Academic Press, New York, 3rd cdition, p. 26.

Liñán, A. and Crespo, A. (1976) An Asymptotic analysis of unsteady diffusion flames for large activation energies. Comb. Sci. and Tech., 14, 95.

Liñán, A. and Williams, F. A. (1993) Fundamental Aspects of Combustion. Oxford University Press, New York, NY, 1st edition, pp. 144-146.

Liñán, A., Orlandi, P., Verzicco, R. and Higuera, J. (1994) Effects of non-unity Lewis numbers in diffusion flames. Proceedings of the 1994 Summer Program: Center for Turbulence Research, NASA Ames/Stanford Univ., pp. 5-18.

Sánchez, A. L., Liñán, A., Williams, F. A. and Balakrishnan, G. (1995) Theory of structures of hydrogen-air diffusion flames. Comb. Sct. and Tech, 110-111, 277.

Sánchez, A. L., Balakrishnan, G., Liñan, A. and Williams, F. A. (1996) Relationships between bifurcation and numerical analyses for ignition of hydrogen-itir diflusion lames, Combust. Flame, 105, 569.

Sánche7, A. L., Liñán, A. and Williams, F. A. (1997) A WKB analysis of radical growth in the hydrogen-air mixing layer, Jounal of Engineeriny Mathematic's, to appear.

Tangirala, V., Seshadri, K.. Treviño, C. and Smooke, M. D. (1991) Analysis of the structure of counterflow hydrogen-air diffusion flames. Progress in Astronanics and Aeronatics. AIA A, 131, 89 .

Treviño, C. and Liñan, A. (1995) Mixing layer ignition of hydrogen, Combust. Flame, 103, 129.

Williams, F. A. (1985) Combustion Theory. Benjamin Cummings, Menlo Park, CA, 2nd edition, pp. $10-12$. 
